\title{
Transfer-matrix scaling from disorder-averaged correlation lengths for diluted Ising systems
}

\author{
S. L. A. de Queiroz ${ }^{a t}$ and R. B. Stinchcombe ${ }^{b}$ \\ ${ }^{a}$ Departamento de Física, Pontifícia Universidade Católica do Rio de Janeiro, \\ Cx. Postal 38071, 22452-970 Rio de Janeiro, RJ, Brazil \\ ${ }^{b}$ Department of Physics, Theoretical Physics, University of Oxford, \\ 1 Keble Road, Oxford OX1 3NP, United Kingdom
}

(October 27, 2018)

\begin{abstract}
A transfer matrix scaling technique is developed for randomly diluted systems, and applied to the site-diluted Ising model on a square lattice in two dimensions. For each allowed disorder configuration between two adjacent columns, the contribution of the respective transfer matrix to the decay of correlations is considered only as far as the ratio of its two largest eigenvalues, allowing an economical calculation of a configuration-averaged correlation length. Standard phenomenological-renormalisation procedures are then used to analyse aspects of the phase boundary which are difficult to assess accurately by alternative methods. For magnetic site concentration $p$ close to $p_{c}$, the extent of exponential behaviour of the $T_{c} \times p$ curve is clearly seen for over two decades of variation of $p-p_{c}$. Close to the pure-system limit, the exactly-known reduced slope is reproduced to a very good approximation, though with non-monotonic convergence. The averaged correlation lengths are inserted into the exponent-amplitude relationship predicted by conformal invariance to hold at criticality. The resulting exponent $\eta$ remains near the pure value $(1 / 4)$ for all intermediate concentrations until it crosses over to the percolation value at the threshold.
\end{abstract}

PACS numbers: $75.10 \mathrm{H}, 75.40 \mathrm{c}, 05.50$ 
The transfer matrix is well known to provide exact solutions for a number of lowdimensional pure systems such as spin models etc [亚. Applied to finite-width strips and combined with finite size scaling [2], it has also proved to be extremely powerful and accurate for non-solvable pure cases, ranging from magnetic systems [3] to walks [4] and quantum Hamiltonians [5]. The application of such "strip-scaling" techniques to random systems has however been extremely limited, despite the enormous interest in such problems as the spin glass, random field and dilute magnets, ceramic superconductors etc. This is because the form so far utilized [6 9] applies the strip scaling to particular realisations of the random system which need not be representative unless appropriate averaging (or self-averaging) is employed, resulting in very large scale computing on extremely long strips and/or many realisations.

Here, we provide a strip scaling approach for random systems, in which the disorder averaging is carried out as one proceeds along the strip, and which therefore does not have the deficiencies noted above. Results reported here extend, and give details of, those contained in a previous Rapid Communication [10]. Our scheme does rely on certain assumptions regarding the dominant contributions to the decay of correlations, which must be spelt out clearly and supported by numerical evidence whenever possible. This is one of our purposes in what follows.

The main numerical application of the technique here is to the two-dimensional sitediluted Ising model [11]. The transfer-matrix descriptions of the special limiting cases of percolation [12] and pure Ising system [13] are well-understood, and are reproduced by the present approach, as we shall see below. On the other hand, several aspects of the behaviour at intermediate dilution are either not known to the same degree of accuracy, or have not been successfully accounted for via transfer-matrix methods. Of the former, we shall focus on the extent of exponential behaviour of the $T_{c} \times p$ curve for magnetic site concentration $p$ close to $p_{c}$, and on the variation of the decay-of-correlations exponent $\eta$ along the critical line; as for the latter, we give results for the reduced slope of the phase boundary close to the pure-system limit, to be compared with an exact solution by Thorpe and McGurn [14].

In the transfer-matrix scaling formulation, the crucial quantity to be considered is the rate of decay of correlations. On a strip, the correlation functions always (i.e. at finite temperatures) decay exponentially for suitably large distances:

$$
<\sigma_{0} \sigma_{R}>\sim \exp (-R / \xi),
$$

with the correlation length $\xi$ being given, for a pure system, by

$$
\xi^{-1}=-\ln \lambda_{2} / \lambda_{1},
$$

where $\lambda_{1}, \lambda_{2}$ are the two largest eigenvalues of the transfer matrix [15]. To obtain this pure system result, the effect of repeated applications of the (same) transfer matrix is investigated, and only leading terms are retained, again in the long-distance limit. In order to have a quantitative understanding of this limit, it is useful to calculate the correlation functions explicitly for the pure Ising model on strips, and compare their actual rate of decay to that predicted by Eqs. 1 and 2. This is illustrated in Fig. 1, where two typical examples are shown. One can see that, for widely differing temperatures and strip widths, the influence of subdominant terms on the correlation decay is always very small and becomes negligible after no more than two lattice spacings. We shall invoke this in what follows. 
For a random system, the decay of thermal correlations for a given realisation of randomness is still given through the iterative product of transfer matrices from one spin column to the next; however, some form of configurational averaging is now necessary. In previous work [6] 9] this was done by generating strips of length $N>>1$ and carrying out the actual transfer-matrix products, so the end result would presumably reflect the properties of a representative sample. While the average free energy per site is unambiguously related to the dominant Lyapunov exponent of the transfer-matrix product [16], the relationship of correlation functions to Lyapunov exponents is much more involved, because the probability distribution of the correlation functions themselves turns out to be rather complex 17,18. It can be shown that the most probable correlation decay is given by the difference between the two largest Lyapunov exponents [17], which constitutes a straightforward generalisation of Eq. 2. This may not be the same as the average correlation decay, which is the experimentally measurable quantity; the distinction between the two becomes especially important when the (effective) interactions fluctuate in sign, as is the case of spin glasses or when random fields are present [17,18]. However, when all interactions are ferromagnetic numerical evidence [19 indicates that, at least for two-dimensional Ising systems, the two quantities yield essentially the same results apart from logarithmic corrections. In what follows, we shall assume this to be the case also when there are absent sites, all non-zero interactions being positive.

The implementation of the schemes just described runs into the usual problems of judging when the strip is sufficiently long, so that the sample can be taken as representative. For diluted systems the additional difficulty would arise of how to avoid the effects of disconnections. In early attempts [20] this was done by introducing a fictitious "weak" bond (of strength $10^{-3}$ that of "normal" ones), linking pairs of sites of which at least one was absent . Numerical results were, however, unsatisfactory.

Here, we perform the averages in a different way. Our starting point is the transfer-matrix formulation of the percolation problem [12]. In this case, which is the zero-temperature limit of diluted magnets, the (geometric) correlation length is correctly given by the largest eigenvalue of a transfer matrix, whose elements are related to the probability that two adjacent site columns (each with occupied and vacant sites) are linked to each other and to the origin. Note that disconnections are altogether avoided from the start, as only column configurations where at least one occupied site is part of the infinite cluster [12 are considered. Unconnected configurations can be discarded, as they just account for conservation of total probability. Were they to be included, the matrix would have 1 as its largest eigenvalue, with the second largest eigenvalue giving the correlation length, in exact correspondence with Eq. 20 [21] Care is thus taken of the correlations between frozen spins in the dilute spin system at absolute zero; as the temperature is raised from zero, each of the possible links represented by the non-zero elements of the geometric transfer matrix is weakened by thermal fluctuations. We propose to take these into account still within the framework of a single matrix, so the exact column-by-column character of the average of disorder configurations will be preserved.

Note however that any approach which directly combines a thermal and disorder averaging (in e.g. multiplying an extended transfer matrix) will be physically incorrect for quenched disorder problems.

So, we must suitably modify each non-zero element of the original matrix; obviously we 
must look at the properties of the spin transfer matrix between the corresponding site column states. As the physical property under investigation is the rate of decay of correlations, we draw on an analogy to the leading contribution towards this in periodic systems: we choose to take the ratio of the two largest eigenvalues of the spin transfer matrix and multiply the corresponding geometric transfer matrix element by it. If $i$ and $j$ are two site column states connected to each other and to the origin, with respective probabilities $P_{i}$ and $P_{j}$, and the spin transfer matrix $T^{i j}$ between these columns has $\lambda_{1}^{i j}$ and $\lambda_{2}^{i j}$ as its largest eigenvalues, the matrix element of the "thermal-geometric" transfer matrix of our formulation is then :

$$
\mathcal{T}_{i j}=\sqrt{P_{i} P_{j}}\left(\lambda_{2}^{i j} / \lambda_{1}^{i j}\right)
$$

The averaged correlation length in this approximation is given by the largest eigenvalue, $\Lambda_{1}$, of $\mathcal{T}$ via

$$
\left(\xi^{-1}\right)_{a v e}=-\ln \Lambda_{1}
$$

The following comments are in order:

(a) As the temperature $T \rightarrow 0$, the two largest eigenvalues of all thermal transfer matrices become degenerate, and Eq. 3 shows that $\mathcal{T}$ reduces to the geometric transfer matrix of Ref. [12], as it should. Thus, the present calculational scheme is asymptotically correct in the low-temperature regime. For other disordered systems this must be true as well, provided that one can start from a suitable transfer-matrix description of the ground-state correlations.

(b) As the concentration $p$ of magnetic sites approaches 1 , the only remaining non-zero element of $\mathcal{T}$ is along the diagonal, connecting two fully occupied columns; denoting by $\lambda_{1}^{\text {pure }}$ and $\lambda_{2}^{\text {pure }}$ the largest eigenvalues of the corresponding thermal transfer matrix,

$$
\Lambda_{1}=\lambda_{2}^{\text {pure }} / \lambda_{1}^{\text {pure }}, \quad p=1
$$

and the pure system limit is correctly obtained.

(c) As befits quenched problems, disorder and thermal aspects are not being averaged together. We represent the effect of thermal fluctuations on each given geometric configuration by the ratio of the respective largest thermal eigenvalues, and the disorder average is performed at a later step, in obtaining the largest eigenvalue of $\mathcal{T}$. Thus we make the analogue of the configurational average of the factor $e^{-1 / \xi}$ in the correlation function (related to its decay between two adjacent columns) and it is indeed the correlation function which is self-averaging.

(d) The procedure outlined contains the approximation that the contribution given by each thermal transfer matrix is truncated and replaced by that of its two largest eigenvalues. Once this has been done, we follow lines analogous to those of Refs. [6, 4, 9] for the calculation of an averaged correlation length in a random system. We are thus (approximately) averaging the two largest Lyapunov exponents, and not the correlation functions. If all thermal transfer matrices, corresponding to distinct disorder configurations, commuted with each other (which is not the case) our ansatz would be identical to that of Refs. [6, [7,9]; note that the probabilistic factors included in our matrix elements would be mirrored in the relative frequency of configurations generated by those methods. On the other hand, the influence of higher-ranking eigenvalues is expected to die out asymptotically, thus minimising the 
truncation effects mentioned above. Having in mind the corresponding results for the pure Ising case, portrayed in Fig 11 above, this does not seem too drastic an assumption.

The results for the critical curve are now presented.

Along the critical line $T_{c}=T_{c}(p)$, the correlation length $\xi$ diverges in the infinite system limit. In phenomenological scaling between strip widths $L$ and $L^{\prime}$ this is where [3] $\xi_{L} / L=$ $\xi_{L^{\prime}} / L^{\prime}$. This condition would normally give a fixed point in a one-parameter space. Here, where two variables $T, p$ occur, we fix $p$ and find the associated critical temperature $T_{c}(p)$ for each $\left(L, L^{\prime}\right)$. As usual, we take periodic boundary conditions along the finite direction of the strips, and $L^{\prime}=L-1$. Sparse-matrix techniques were used for the diagonalisation of the thermal transfer matrices. We have obtained the approximate phase diagrams using phenomenological scaling with $L$ up to 7 . For each temperature and concentration at the next step, $L=8$, one would need to take into account nearly 32,000 configurations of connected pairs of columns and extract the two largest thermal eigenvalues of the respective transfer matrices; though symmetry considerations can reduce the number of distinct configurations involved, analysis of a few selected points convinced us that no additional insight would be gained this way. Data from $L=5,6$ and 7 scalings are shown in Fig. 2, together with an interpolated curve incorporating the exact values of $T_{c}(p=1)$ and of the limiting slope at $p=1$, as well as the best estimate for the percolation threshold $p_{c}=0.592745 \pm 0.000002$ [22]. The overall picture is similar to those provided by simple analytic scaling approaches 111] and Monte Carlo simulations [25].

At the extreme points $p=p_{c}, T_{c}=0$ and $p=1, T_{c}=T_{c}(p=1)$ it is known (respectively from Refs. [12] and [13]) that the approach of strip-scaling fixed points to the exact critical parameters is monotonic as $L$ grows; as remarked in (a) and (b) above, these sequences are automatically reobtained in our scheme. For intermediate concentrations, the same uniform convergence does not seem to be present, as curves from successive scalings cross each other. This is almost certainly related to the approximations involved in the truncations referred to above. However, the curves are very close to each other, and to the interpolated phase boundary. Thus, although the truncation effects do not die away with increasing $L$ (and there is no a priori reason they should do so), they are most probably of a quantitatively limited nature.

We now turn to results for the reduced slope $\left(1 / T_{c}(1)\right)\left(d T_{c}(p) / d p\right)_{p=1}$ of the critical curve at the pure limit, which is known exactly [14]. Close to $p=1$ we were able to reach $L=11$ by using a truncated basis of connected states, consisting only of pairs of adjacent columns with at most two vacant sites overall. We checked the consistency of results thus obtained against those from the full basis (for $L \geq 6$ ) and from a basis with up to four vacant sites per two-column configuration (for $L \geq 8$ ). We made use of analytical expressions linking $d T_{c}(p) / d p$ with $\partial \xi / \partial T$ and $\partial \xi / \partial p$, through the condition $\xi_{L} / L=\xi_{L^{\prime}} / L^{\prime}$, which defines the critical line. Both partial derivatives can be evaluated by first-order perturbation theory instead of numerically; thus, calculations were performed very close to $p=1\left(1-p=10^{-8}\right.$ was typical), without loss of accuracy .

Results from $(L, L-1)$ scalings up to $L=11$ are given in Table 1 , which complements the corresponding table of Ref. [10]. The sudden discontinuity when one goes from $\left(L, L^{\prime}\right)=(7,6)$ to $(8,7)$ is similar to what takes place for the fixed point of percolation between $\left(L, L^{\prime}\right)=$ $(4,3)$ and $(5,4)$ [12,21], where convergence is interrupted.The extrapolation quoted in Table 1 was obtained by taking the last four points of the sequence, and searching for the value of 
$\psi$ such that the plot of those data against $L^{-\psi}$ was the best possible straight line (see the work of Derrida and Stauffer in Ref. [12]). Here, one has $\psi \simeq 2.0$. The exact slope is due to Thorpe and McGurn [14. The behaviour of the critical curve near the percolation threshold is determined by a crossover exponent $\phi$ giving the power-law dependence of $e^{-2 J / T_{c}(p)}$ on $\left(p-p_{c}\right)$. As $\phi$ is known exactly to be 1 [23], this can provide another test of the quality of the strip scaling results.

At low temperatures, our ansatz is asymptotically exact. Care must be taken, however, with the spin degrees of freedom of occupied sites that are not directly connected to the origin [12,21]. With periodic boundary conditions, these may occur for $L \geq 4$. No matter whether they belong to finite clusters or are connected by a path that goes forwards before turning backwards to the origin, such sites do not contribute to the spread of thermal correlations along the backbone of the infinite cluster (they do, on the other hand, matter for the statistics of geometric configurations, so their probabilistic weight must be correctly included when counting the latter). In the corresponding thermal transfer matrices, the occupied unconnected sites are then treated as if they were absent. This is done, of course, for all temperatures and concentrations, but has well-marked effects in the low-temperature region. We have found that, if unconnected spins are not frozen out, the $T_{C} \times p$ curves for $L \geq 4$ develop an unphysical overhang at $T \rightarrow 0, p<p_{c}$ before homing in towards the correct $p_{c}$ at $T=0$. Once these corrections are incorporated, we get the correct vertical approach to $p_{c}$ in the $T-p$ plane.

Slow convergence problems put a limit to the lowest temperatures within reach of investigation; this is usually of order $10 \%$ of the critical temperature for the pure system, $T_{c}(1)$; for the smallest strip widths, $l=2-4$ we get to $\sim 0.06 T_{c}(1)$. As the phase boundary is vertical in that neighbourhood, the corresponding values of $p-p_{c}$ are $\sim 10^{-5}$. This is enough to see the exponential behaviour, with $\phi=1$ along more than two orders of magnitude of variation in $p-p_{c}$. In Fig. 3 we show, for $L=5,6$ and 7 , the quantity

$$
C_{L} \equiv \frac{\exp \left(-2 J / T_{c}(p)\right)}{p-p_{c}(L)}
$$

against $\ln \left(p-p_{c}(L)\right)$ (where $p_{c}(L)$ is the fixed point of phenomenological renormalisation, and $T_{c}(p)$ is obtained from our ansatz, both for strips of widths $L$ and $\left.L-1\right)$. For $L=6$ and 7 , the region in which $C_{L}$ is approximately constant goes from $p-p_{c}(L) \simeq 4 \times 10^{-5}$ to $\simeq 6 \times 10^{-3}$. The approximate values at which the $C_{L}$ become stable are: $6.1,3.8$ and 2.3 respectively for $L=5,6$ and 7 . It does not seem possible to obtain a reasonable estimate of the limiting value of this coefficient, $C_{\infty}$ from extrapolation of this sequence. For bond dilution, a value for comparison is [24] $2 \ln 2=1.386 \ldots$. A heuristic generalisation for site dilution (see [11], pg. 190) would lead to $C_{\infty}=\left(1 / p_{c}\right) \ln 2=1.169 \ldots$ The interpolated curve used in Fig.2 has $C \sim 4$, for consistency with corresponding data of Ref. [10]; for purposes of overall comparison of the shape of the phase diagram, the difference relative to the presumed value $\simeq 1.169$ is not relevant.

It must be remarked that the region covered in Fig. 3 is very difficult to reach e.g. in Monte-Carlo simulations, as one is at concentrations extremely close to criticality. For comparison, the lowest $p$ used in recent work [25] corresponds to $p-p_{c} \simeq 7 \times 10^{-3}$. From the data depicted above, it seems safe to conclude that the exponential behaviour is dominant at least up to a critical temperature of order $T_{c} / J \sim 0.7$. 
Conformal invariance [26] allows one to extract additional information from strip scaling, via the relationship between correlation-length amplitudes on a strip of width $L$ at criticality and the decay-of-correlations exponent $\eta$ :

$$
\eta=L / \pi \xi\left(T_{c}\right) .
$$

In the present case, one has two questions to answer: first, whether conformal invariance still is valid for random systems: second, if so, how to define the correlation length which enters Eq.7. As for the former, transfer-matrix [9] and field-theoretical results [27] indicate the affirmative, provided that one considers averages over disorder. The latter is more involved, with the two most obvious candidates being the correlation decay factors related to the "most probable" and "average" correlation functions [16,17]. Based on numerical evidence for the bond-disordered two-dimensional Ising system [19], we assume that for dilution the distinction between results coming from either definition amounts at most to logarithmic corrections. Thus, we shall use the average correlation length given by Eq.⿴囗十 which, as remarked above, is closely related to an average of the two largest Lyapunov exponents, i.e., the most probable correlation decay.

In Fig. (1) we display the values of $\eta$ along the approximate critical curves. As can be seen, behaviour is not uniform with $L$; however, the overall trend for the exponent is to keep an approximately constant value close to that of the pure Ising model, $\eta_{I}=1 / 4$, and drop towards the percolation value $\eta_{p}=5 / 24$ close to $p_{c}$.

The above-mentioned trend becomes much more apparent, and uniform with $L$, when one considers the variation of $\eta$ along the interpolated critical curve. Fig.5 illustrates this. Note the difference between vertical scales in Figs. 9 and 5. Recall from Fig.? that the approximate and interpolated curves are very close in $T-p$ space. This shows that the averaged correlation length is very sensitive to slight variations in the parameters. In both figures, it is apparent that the values of $\eta$ at $p=p_{c}, p=1$ approach the exact values as $L$ increases. Again, the two special cases of the pure Ising model and percolation have been treated previously [12,13], and our results for these limits coincide with those already obtained.

The behaviour depicted above is to be compared with current field theory results for random Ising models in two dimensions [27 29]. The theories agree in predicting that the specific heat singularity is of $\ln \ln \left(T-T_{c}\right)$ type rather than the $\ln \left(T-T_{c}\right)$ of the pure case. But one group of theories [27] predicts that the asymptotic pair correlation is as in the pure case $\left(\left\langle\sigma_{0} \sigma_{r}\right\rangle \sim r^{-\eta}, \eta=1 / 4\right)$ while the other [28] concludes that the correlation has the form $\left\langle\sigma_{0} \sigma_{r}>\sim e^{-A(\ln r)^{2}}\right.$.

Our results clearly support the first class of predictions, and show no sign of the huge change of $\eta$ expected if the second prediction were correct.

Large Monte Carlo simulations on the random-bond Ising model at a particular (selfdual) concentration [29] also provide indirect evidence in favour of the first class of results. An evaluation of the susceptibility exponent $\gamma$ is taken together with scaling relations to infer a value for $\eta$, which is close to the pure value $1 / 4$. A direct evaluation of $\eta$, again pointing to the pure result, has also been obtained [9] for the same random bond Ising model by strip scaling on long realisations of the random system. Very recent Monte Carlo results for the correlation functions at criticality also support this view [30].

In conclusion, application of the calculational scheme just described to the site-diluted 
Ising model gives reliable and fairly accurate results, though convergence towards the exact values as strip width $L$ increases seems to be generally non-uniform. Our data for the exponent $\eta$ at intermediate concentrations give clear evidence in favour of one of the competing classes of field-theoretic treatments [28,27,29, 30.

A straightforward extension of the method to the random-field Ising model in two dimensions gives results for the averaged correlation length which are qualitatively similar to those predicted from the two largest Lyapunov exponents [31]. These, in turn, seem to differ appreciably from the correlation lengths obtained directly from direct fits of the averaged correlation decay. As remarked above, cases such as this where frustrations are present tend to much subtler than when all correlations are ferromagnetic. We defer a detailed analysis of this point to a forthcoming publication.

\section{ACKNOWLEDGMENTS}

SLAdQ would like to thank the Department of Theoretical Physics at Oxford, where this work was initiated, for the hospitality, and the cooperation agreement between Academia Brasileira de Ciências and the Royal Society for funding a later visit to Oxford. Research of SLAdQ is partially supported by the Brazilian agencies Ministério da Ciência e Tecnologia, Conselho Nacional de Desenvolvimento Científico e Tecnológico and Coordenação de Aperfeiçoamento de Pessoal de Ensino Superior. 


\section{REFERENCES}

* $\quad$ Electronic address: sldq@fis.puc-rio.br

[1] See for example R.J. Baxter, Exactly Solved Models in Statistical Mechanics (Academic, New York, 1982).

[2] M.E. Fisher, in Proceedings of the "Enrico Fermi" International School of Physics, Varenna, 1970, Course No. 51 edited by M.S.Green (Academic, New York, 1971).

[3] M.N. Barber, in Phase Transitions and Critical Phenomena Vol. 8, edited by C. Domb and J.L. Lebowitz (Academic, New York, 1983); M.P. Nightingale, in Finite Size Scaling and Numerical Simulations of Statistical Systems, edited by V. Privman (World Scientific, Singapore, 1990).

[4] B. Derrida, J. Phys A14, L5 (1981).

[5] M. Henkel, in Finite Size Scaling and Numerical Simulations of Statistical Systems, edited by V. Privman (World Scientific, Singapore, 1990) .

[6] H.-F. Cheung and W.L. McMillan, J. Phys. C16, 7027 (1983); ibid. C16, 7033 (1983).

[7] D.A. Huse and I.M. Morgenstern, Phys. Rev. B32, 3032 (1985).

[8] U. Glaus, Phys. Rev. B34, 3203 (1986).

[9] U. Glaus, J. Phys. A20, L595 (1987).

[10] S. L. A. de Queiroz and R. B. Stinchcombe, Phys. Rev. B46, 6635 (1992).

[11] See for example R.B. Stinchcombe, in Phase Transitions and Critical Phenomena Vol. 7, edited by C. Domb and J.L. Lebowitz (Academic, New York, 1983).

[12] B. Derrida and L. de Seze, J. Phys. (Paris) 43, 475 (1982); B. Derrida and D. Stauffer, ibid. 46, 1623 (1985) .

[13] M.P. Nightingale, J. Appl. Phys. 53, 7927 (1982).

[14] M.F. Thorpe and A. McGurn, Phys. Rev. B20, 2142 (1979).

[15] C. Domb, Adv. Phys. 9, 149 (1960) .

[16] A. Crisanti, G. Paladin and A. Vulpiani, Products of Random Matrices in Statistical Physics (Springer-Verlag, Berlin, 1991).

[17] A. Crisanti, S. Nicolis, G. Paladin and A. Vulpiani, J. Phys. A23, 3083 (1990).

[18] B. Derrida, Phys. Rep. 103, 29 (1984).

[19] S.L.A. de Queiroz and R.B. Stinchcombe (to be published, 1994)

[20] K.K. Kaski, D. Phil Thesis, University of Oxford (unpublished, 1980).

[21] B. Derrida and J. Vannimenus, J. Phys. Lett. (Paris) 41, L-473 (1980).

[22] R.M. Ziff and B. Sapoval, J. Phys. A19, L1169 (1986) .

[23] A. Coniglio, Phys.Rev.Lett. 46, 250 (1981)

[24] E. Domany, J. Phys. C11, L337 (1978)

[25] A.J.F. de Souza and F.G. Brady Moreira, Europhys. Lett. 17, 491 (1992)

[26] J. L. Cardy, in Phase Transitions and Critical Phenomena, edited by C. Domb and J.L. Lebowitz (Academic, New York, 1987), vol. 11 .

[27] A.W.W. Ludwig, Nucl. Phys. B330, 639 (1990).

[28] Vik.S. Dotsenko and Vl.S. Dotsenko, J. Phys. C15, 495 (1982)

[29] J.-S. Wang, W. Selke, Vl.S. Dotsenko and V.B. Andreichenko, Physica A164, 221 (1990).

[30] A.L. Talapov and L.N. Shchur, preprint hep-lat/9404002 (1994)

[31] D. Moore, R.B. Stinchcombe and S.L.A. de Queiroz, work in progress. 


\section{TABLES}

TABLE I. Reduced slope at $p=1$

$\begin{array}{cc}L / L^{\prime} & \frac{1}{T_{c}(1)}\left(\frac{d T_{c}(p)}{d p}\right)_{p=1} \\ 3 / 2 & 1.4461 \\ 4 / 3 & 1.4765 \\ 5 / 4 & 1.5032 \\ 6 / 5 & 1.5165 \\ 7 / 6 & 1.5223 \\ 8 / 7 & 1.4944 \\ 9 / 8 & 1.5015 \\ 10 / 9 & 1.5065 \\ 11 / 10 & 1.5101 \\ \text { Extrapolated } & 1.53 \pm 0.015 \\ \text { Exact } & 1.565\end{array}$




\section{FIGURES}

FIG. 1. Semi-logarithmic plot of correlation function $\left\langle\sigma_{0} \sigma_{R}\right\rangle$ (squares) against distance $R$ for the pure Ising model on strips of width $L$ of a square lattice. Values of $\xi$ in lattice spacing units, are as given by Eq. 2.Straight lines have slope $-1 / \xi . T_{c}$ is the critical temperature of the two-dimensional lattice.

FIG. 2. Approximate phase diagram from $(L, L-1)$ scalings for $L=5,6$ and 7 and from interpolation between exact limits.

FIG. 3. Exponential behaviour of $T_{c}$ against $p$ close to $p_{c}$. See Eq. 6 for definition of $C_{L}$.

FIG. 4. Correlation exponent $\eta$ from correlation-length amplitudes, along approximate $(L, L-1)$ critical curves. Black squares are at $(0.59275,5 / 24)$ and $(1,1 / 4)$.

FIG. 5. Correlation exponent $\eta$ from correlation-length amplitudes, along interpolated critical curve. Black squares are at $(0.59275,5 / 24)$ and $(1,1 / 4)$. 


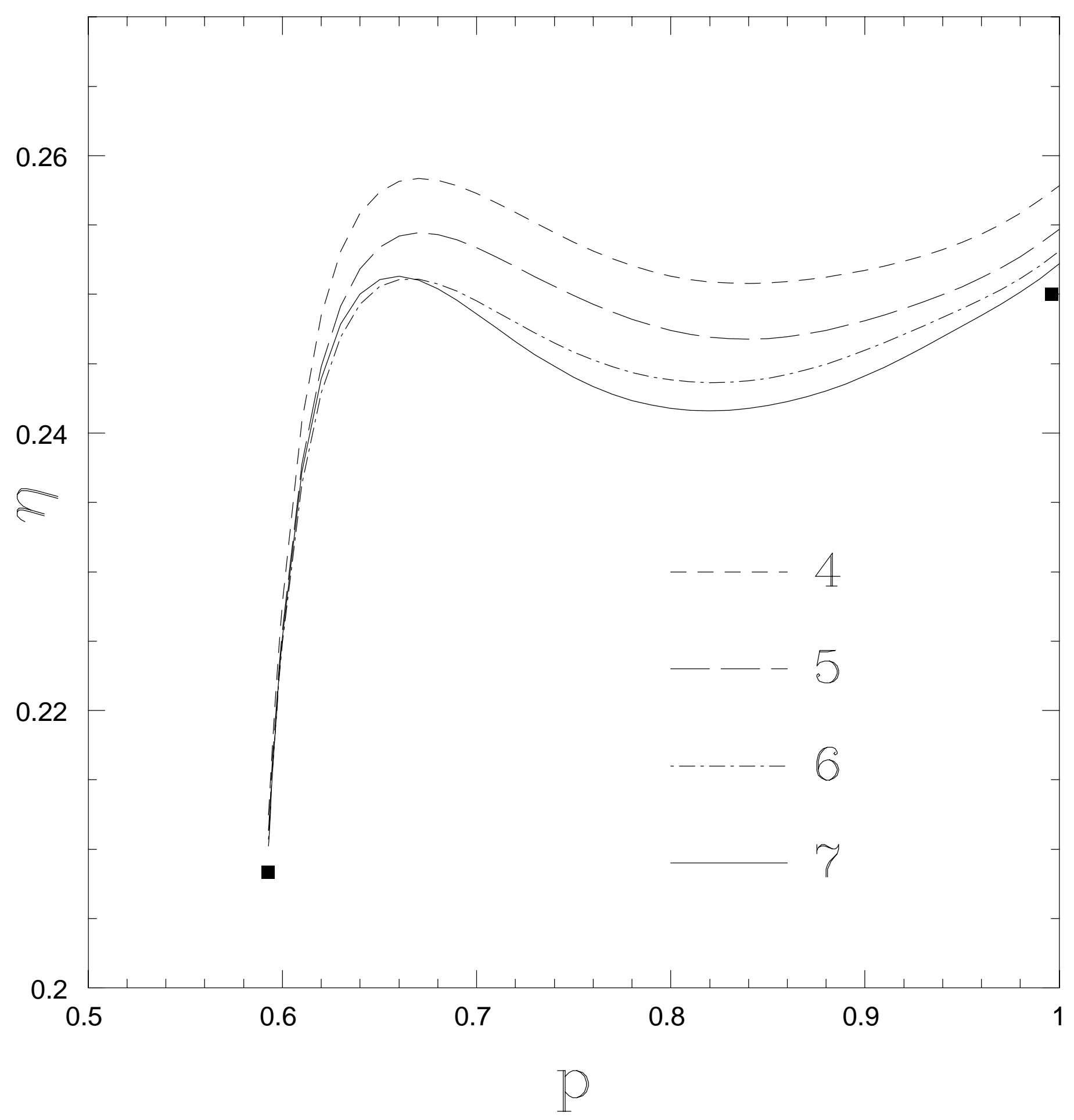




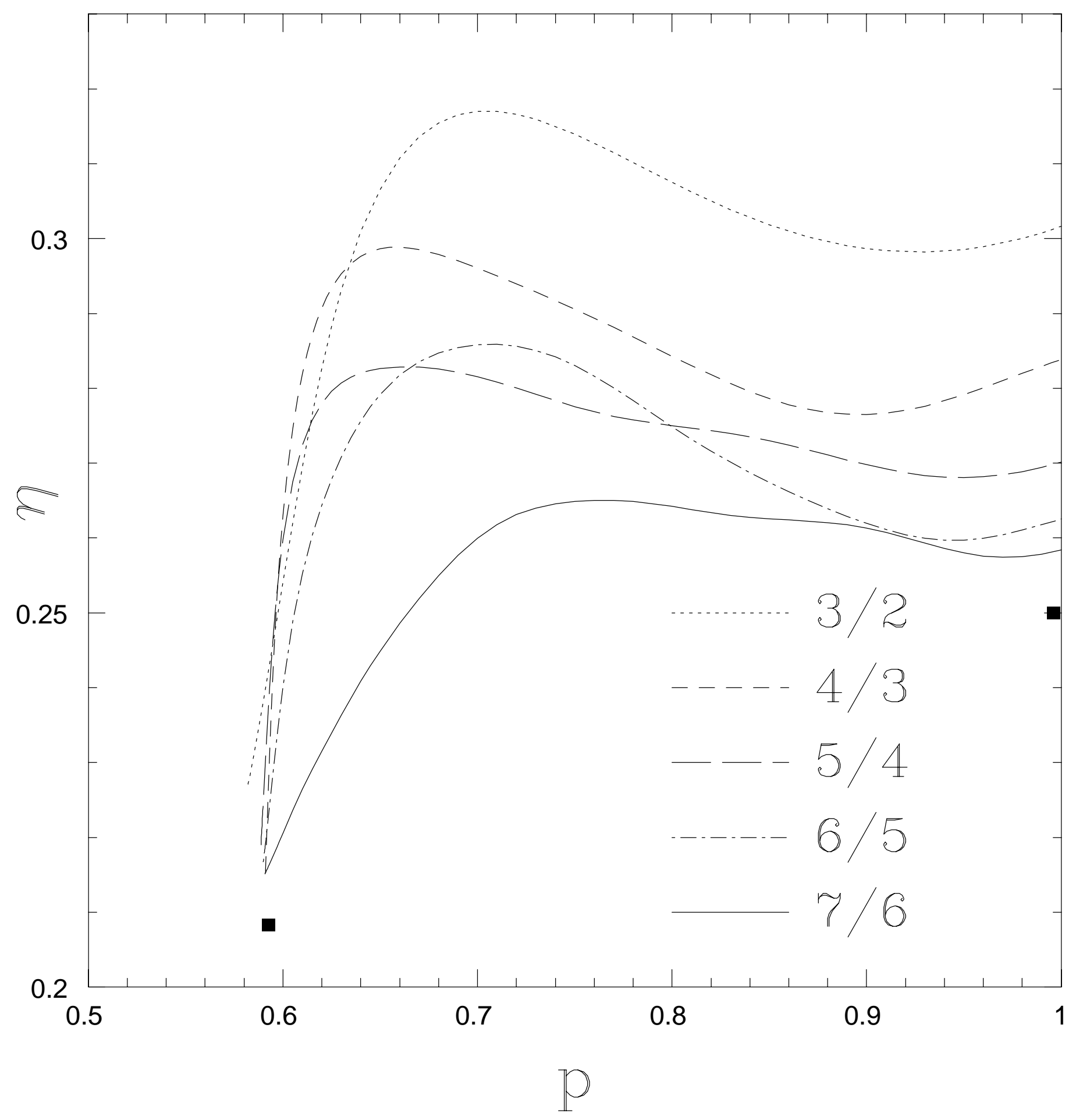




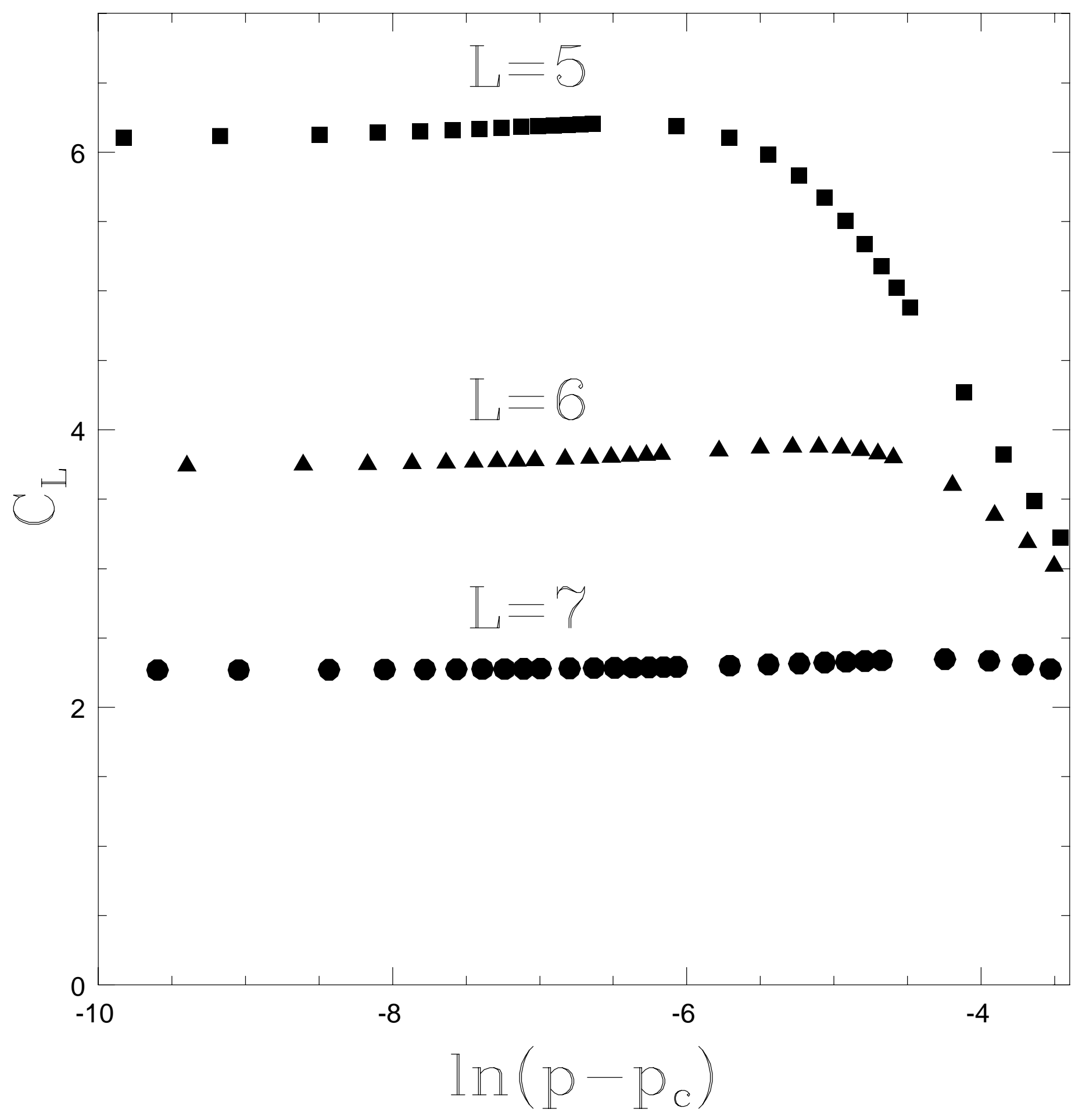




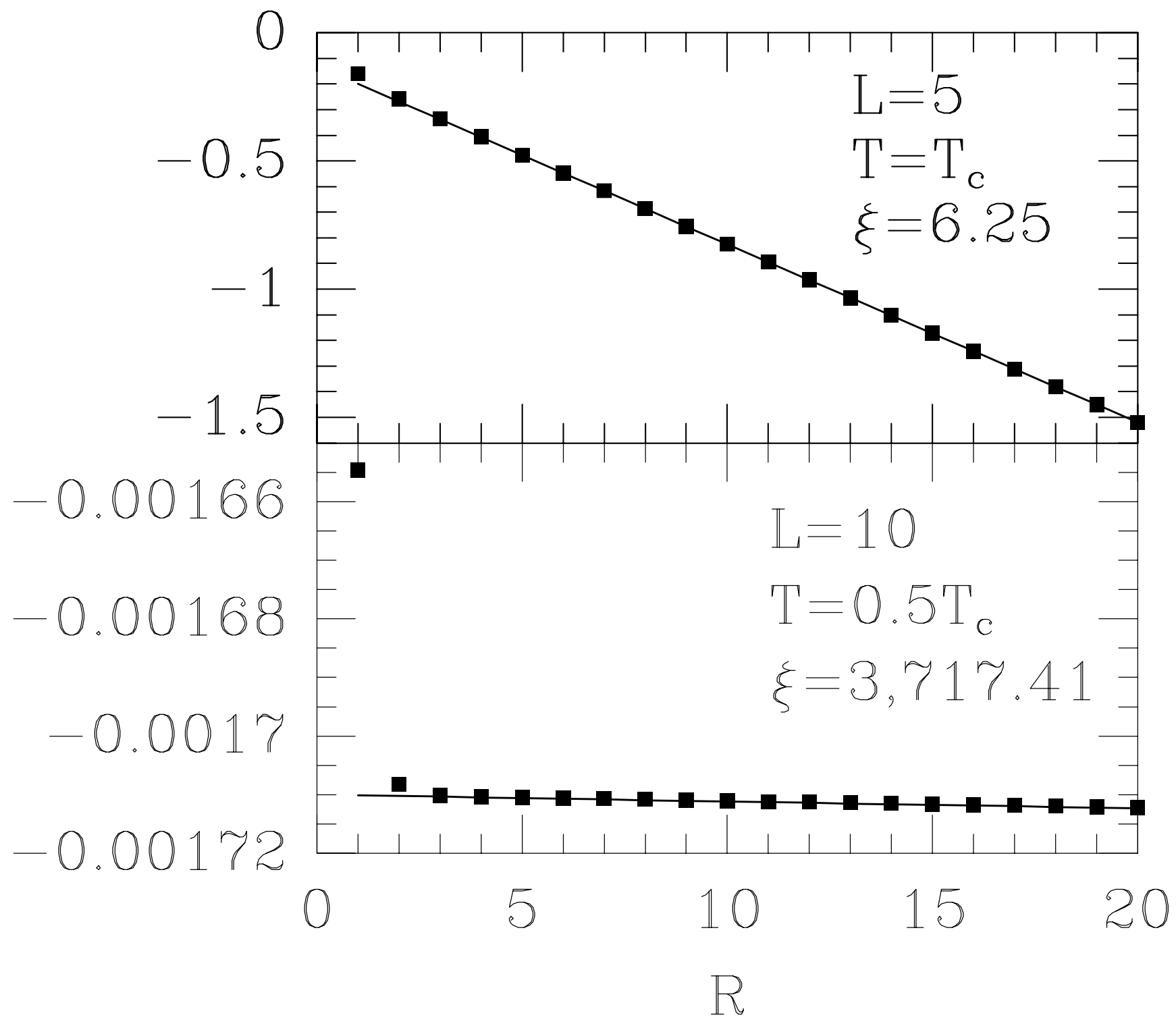




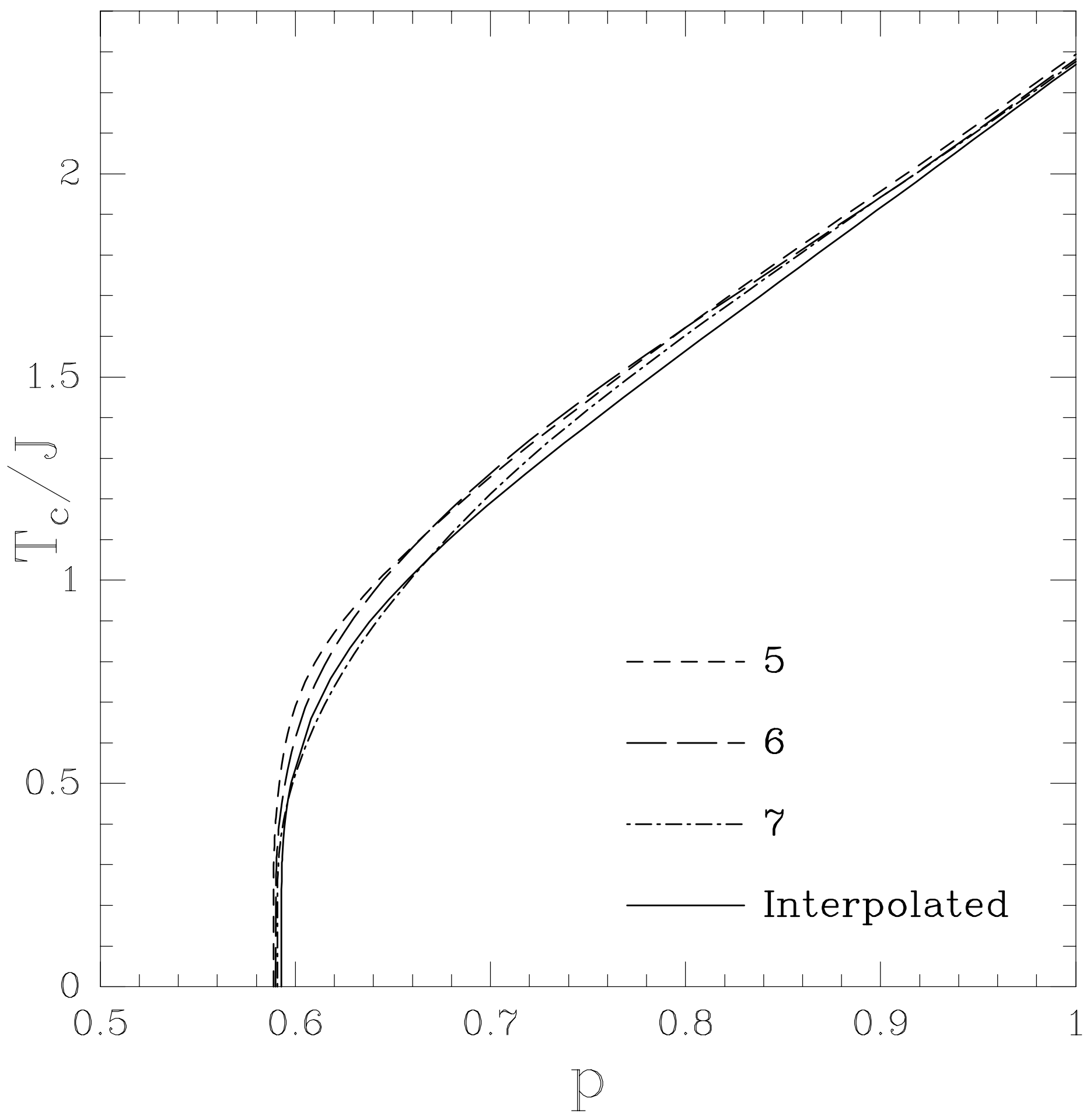

